\title{
GEOMETRY OF POINTWISE CR-SLANT WARPED PRODUCTS IN KAEHLER MANIFOLDS
}

\author{
BANG-YEN CHEN, SIRAJ UDDIN, AND FALLEH R. AL-SOLAMY
}

\begin{abstract}
We call a submanifold $M$ of a Kaehler manifold $\tilde{M}$ a pointwise $C R$-slant warped product if it is a warped product, $B \times_{f} N_{\theta}$, of a CR-product $B=N_{T} \times N_{\perp}$ and a proper pointwise slant submanifold $N_{\theta}$ with slant function $\theta$, where $N_{T}$ and $N_{\perp}$ are complex and totally real submanifolds of $\tilde{M}$. We prove that if a pointwise CR-slant warped product $B \times_{f} N_{\theta}$ with $B=N_{T} \times N_{\perp}$ in a Kaehler manifold is weakly $\mathfrak{D}^{\theta}$-totally geodesic, then it satisfies

$$
\|\sigma\|^{2} \geq 4 s\left\{\left(\csc ^{2} \theta+\cot ^{2} \theta\right)\left\|\nabla^{T}(\ln f)\right\|^{2}+\left(\cot ^{2} \theta\right)\left\|\nabla^{\perp}(\ln f)\right\|^{2}\right\},
$$

where $N_{T}, N_{\perp}$, and $N_{\theta}$ are complex, totally real and proper pointwise slant submanifolds of $\tilde{M}$, respectively, and $s=\frac{1}{2} \operatorname{dim} N_{\theta}$. In this paper we also investigate the equality case of the inequality. Moreover, we give a non-trivial example and provide some applications of this inequality.
\end{abstract}

\section{INTRODUCTION}

A warped product $N_{1} \times_{f} N_{2}$ of two Riemannian manifolds $\left(N_{1}, g_{1}\right)$ and $\left(N_{2}, g_{2}\right)$ is the product manifold $N_{1} \times N_{2}$ equipped with the warped product metric

$$
g=g_{N_{1}}+f^{2} g_{N_{2}},
$$

where $f: N_{1} \rightarrow \mathbb{R}^{+}$is a positive smooth function on $N_{1}$. The function $f$ is called the warping function [1, 12. When the warping function $f$ is constant, $M=N_{1} \times_{f} N_{2}$ is simply a Riemannian product. It is known that, for a vector field $X$ on $N_{1}$ and a vector field $Z$ on $N_{2}$, we have

$$
\nabla_{X} Z=\nabla_{Z} X=X(\ln f) Z
$$

where $\nabla$ is the Levi-Civita connection on $M$. Further, it is well-known that $N_{1}$ is totally geodesic and $N_{2}$ is totally umbilical in $N_{1} \times_{f} N_{2}$ (see e.g. [1, 6, 7, 9]).

A submanifold $M$ of a Kaehler manifold $\tilde{M}$ is called a $C R$-product if it is locally the Riemannian product $N_{T} \times N_{\perp}$ of a complex submanifold $N_{T}$ and a totally real submanifold $N_{\perp}$ of $\tilde{M}$ (see [2]). B.-Y. Chen had determined CR-products in

2010 Mathematics Subject Classification. 53C15, 53C40, 53C42, 53B25.

Key words and phrases. CR-product; CR-slant warped product; slant submanifold; pointwise slant submanifold; totally real submanifold; complex submanifold; inequality.

This project was funded by the Deanship of Scientific Research (DSR), King Abdulaziz University, Jeddah, Saudi Arabia under grant no. (KEP-PhD-88-130-38). The authors, therefore, acknowledge with thanks DSR's technical and financial support. 
complex space forms in [2, 3]. In [6] Chen proved that there do not exist warped product submanifolds of the form $N_{\perp} \times_{f} N_{T}$ in any Kaehler manifolds $\tilde{M}$, where $N_{T}$ is a complex submanifold and $N_{\perp}$ is a totally real submanifold of $\tilde{M}$. On the other hand, he introduced the notion of $C R$-warped products in $\tilde{M}$ as warped product submanifolds of the form $N_{T} \times_{f} N_{\perp}$. Since then there are many results on warped product submanifolds studied by many geometers (see, for instance, [7, 8, 11, 12, 15, 17, 18, 20, 21, 22, 23, 24, 25, 26]). In particular, B. Sahin in [20] proved that there are no warped product submanifolds of the form $N_{T} \times_{f} N_{\lambda}$ in Kaehler manifolds, where $N_{\lambda}$ is a slant submanifold with slant angle $\lambda \in\left(0, \frac{\pi}{2}\right)$. He also studied warped product submanifolds of the form $N_{T} \times_{f} N_{\theta}$ in [22], where $N_{\theta}$ is a pointwise slant submanifold, instead of a slant submanifold. Sahin named such submanifolds pointwise semi-slant warped product submanifolds. Also, Sahin proved in 21] that there do not exist warped product submanifolds of the form $N_{\perp} \times_{f} N_{\lambda}$ in Kaehler manifolds such that $N_{\perp}$ is totally real and $N_{\lambda}$ is a proper slant. Further, he also studied in [21] warped product hemi-slant submanifolds which are of the form $N_{\lambda} \times_{f} N_{\perp}$. Warped product hemi-slant submanifolds were extended to warped product pointwise hemi-slant submanifolds and studied in [23, 26].

In this paper, we introduce and investigate a more general family of warped product submanifolds, called pointwise CR-slant warped products, in Kaehler manifolds, which are of the form $B \times{ }_{f} N_{\theta}$, where $B=N_{T} \times N_{\perp}$ is a CR-product and $N_{\theta}$ is a proper pointwise slant submanifold of $\tilde{M}$.

Our main purpose is to establish the following sharp inequality:

$$
\|\sigma\|^{2} \geq 4 s\left\{\left(\csc ^{2} \theta+\cot ^{2} \theta\right)\left\|\nabla^{T}(\ln f)\right\|^{2}+\left(\cot ^{2} \theta\right)\left\|\nabla^{\perp}(\ln f)\right\|^{2}\right\},
$$

for weakly $\mathfrak{D}^{\theta}$-totally geodesic, pointwise CR-slant warped products in a Kaehler manifold, where $s=\frac{1}{2} \operatorname{dim} N_{\theta}$ and $\|\sigma\|$ is the norm of the second fundamental form.

The paper is organized as follows: In Section 2, we give basic definitions and formulas. Definition and a non-trivial example of CR-slant warped products are given in Section 3. In Section 4, we provide useful lemmas and propositions for the proof of our main theorem. The proof of inequality $\sqrt{1.2}$ and the study of the equality case of $(1.2)$ are given in Section 5. The last section provides several applications of the main theorem.

\section{Preliminaries}

A $2 m$-manifold $\tilde{M}^{2 m}$ with an almost complex structure $J$ and a Riemannian metric $\langle$,$\rangle is said to be a Kaehler manifold if it satisfies (see e.g. [4, 10, 27])$

$$
J^{2}=-I, \quad\langle J U, J V\rangle=\langle U, V\rangle, \quad\left(\tilde{\nabla}_{U} J\right) V=0
$$

for vector fields $U, V$ tangent to $\tilde{M}$, where $\tilde{\nabla}$ is the Levi-Civita connection on $\tilde{M}$ and $I$ is the identity transformation.

Let $M$ be a submanifold of $\tilde{M}^{2 m}$. Denote by $\Gamma(T M)$ the Lie algebra of vector fields on $M$ and let $\Gamma\left(T^{\perp} M\right)$ denote the set of vector fields normal to $M$. The 
formulas of Gauss and Weingarten are given respectively by

$$
\begin{gathered}
\tilde{\nabla}_{X} Y=\nabla_{X} Y+\sigma(X, Y), \\
\tilde{\nabla}_{X} \xi=-A_{\xi} X+D_{X} \xi,
\end{gathered}
$$

for $X, Y \in \Gamma(T M)$ and $\xi \in \Gamma\left(T^{\perp} M\right)$, where $D$ denotes the normal connection of the normal bundle $T^{\perp} M$ and $A$ is the shape operator of $M$. The second fundamental form $\sigma$ and the shape operator $A$ of $M$ are related by

$$
\langle\sigma(X, Y), \xi\rangle=\left\langle A_{\xi} X, Y\right\rangle,
$$

where $\langle$,$\rangle denotes the inner products on M$ and $\tilde{M}$ with respect to their metrics.

For any vector field $X$ tangent to $M$, we put

$$
J X=P X+F X,
$$

where $P X$ is the tangential component and $F X$ is the normal component of $J X$.

For any vector field $\xi$ normal to the submanifold $M$, we put

$$
J \xi=t \xi+f \xi,
$$

where $t \xi$ and $f \xi$ are the tangential and normal components of $J \xi$, respectively.

A submanifold $M$ of a Kaehler manifold $\tilde{M}^{2 m}$ is called a complex submanifold if $J\left(T_{x} M\right) \subset T_{x} M, \forall x \in M$, where $T_{x} M$ denotes the tangent space of $M$ at $x \in M$. It is called totally real if $J\left(T_{x} M\right) \subset T_{x}^{\perp} M, \forall x \in M$, where $T_{x}^{\perp} M$ is the normal space of $M$ at $x \in M$ (see [14]).

Definition 2.1. A submanifold $M$ of a Kaehler manifold is called pointwise slant if for each $x \in M$, the Wirtinger angle $\theta(X)$ between $J X$ and $T_{x} M$ is independent of the choice of $X \in T_{x} M \backslash\{0\}$. The function $\theta: T M \backslash\{0\} \rightarrow \mathbb{R}$ is called the slant function of the pointwise slant submanifold $M$ (see [13, 16, 19]).

A pointwise slant submanifold is called a slant submanifold if its slant function $\theta$ is globally constant; such $\theta$ is called the slant angle of the slant submanifold (see [4, 5]). Clearly, the same definitions apply to submanifolds in almost Hermitian manifolds. Obviously, complex and totally real submanifolds are slant submanifolds with slant angle $\theta=0$ and $\theta=\frac{\pi}{2}$, respectively,

Definition 2.2. A pointwise slant submanifold $M$ of a Kaehler manifold $\tilde{M}$ is called proper if its slant function $\theta$ satisfies $0<\theta<\frac{\pi}{2}$. Thus proper pointwise slant submanifolds are neither complex nor totally real.

From Lemma 2.1 of [13, we know that a submanifold $M$ of an almost Hermitian manifold $\tilde{M}$ is a pointwise slant submanifold if and only if

$$
P^{2} X=-\left(\cos ^{2} \theta\right) X,
$$

for some real-valued function $\theta$ defined on $M$. The following relations are immediate consequences of 2.5 :

$$
\begin{aligned}
& \langle P X, P Y\rangle=\cos ^{2} \theta\langle X, Y\rangle, \\
& \langle F X, F Y\rangle=\sin ^{2} \theta\langle X, Y\rangle
\end{aligned}
$$


for $X, Y \in \Gamma(T M)$. The next relation for pointwise slant submanifolds of an almost Hermitian manifold follows easily from (2.1) and 2.5):

$$
t F X=-\sin ^{2} \theta X, \quad f F X=-F P X
$$

for $X \in \Gamma(T M)$.

Notation 2.3. Let $\mathbb{R}^{n}$ denote the Cartesian $n$-space and let

$$
\mathbb{E}^{2 m}=\left\{\left(x_{1}, \ldots, x_{m}, y_{1}, \ldots, y_{m}\right): x_{i}, y_{i} \in \mathbf{R}, i=1, \ldots, n\right\}
$$

be the Euclidean $2 m$-space with natural coordinates $\left(x_{1}, \ldots, x_{m}, y_{1}, \ldots, y_{m}\right)$ and with the standard Euclidean metric $g_{0}=\sum_{i=1}^{m}\left(d x_{i}^{2}+d y_{i}^{2}\right)$. Consider the almost complex structure $J$ on $\mathbb{E}^{2 m}$ given by

$$
J\left(x_{1}, \ldots, x_{m}, y_{1}, \ldots, y_{m}\right)=\left(-y_{1}, \ldots,-y_{m}, x_{1}, \ldots, x_{m}\right) .
$$

Then the complex Euclidean $m$-space $\mathbb{C}^{m}=\left(\mathbb{E}^{2 m}, J\right)$ is a flat Kaehler manifold.

\section{Pointwise CR-Slant Warped Products: Definition And EXAmple}

Now, we provide the following definitions.

Definition 3.1. Let $M$ be a submanifold of a Kaehler manifold $(\tilde{M}, \tilde{g}, J)$. A distribution $\mathfrak{D}$ on $M$ is called a complex distribution (resp., a totally real distribution) if $J(\mathfrak{D}) \subset \mathfrak{D}\left(\right.$ resp., $\left.J(\mathfrak{D}) \subset T^{\perp} M\right)$.

Definition 3.2. A distribution $\mathfrak{D}$ on $M$ is called pointwise slant if for each given point $p \in M$ and any unit vector $X \in \mathfrak{D}_{p}$, the angle $\theta(X)$ between $J X$ and $\mathfrak{D}_{p}$ is independent of the choice of $X$. The function $\theta$ is called the slant function. In particular, if the angle $\theta(X)$ between $J X$ and $\mathfrak{D}_{p}$ is also independent of the choice of point $p \in M$, then the distribution $\mathfrak{D}$ is called a slant distribution.

Definition 3.3. Let $M$ be a submanifold of a Kaehler manifold $\tilde{M}$. Assume that there exist three integrable distributions $\mathfrak{D}^{T}, \mathfrak{D}^{\perp}$, and $\mathfrak{D}^{\theta}$ on $M$ such that

$$
T M=\mathfrak{D}^{T} \oplus \mathfrak{D}^{\perp} \oplus \mathfrak{D}^{\theta},
$$

where $\mathfrak{D}^{T}$ is a complex distribution, $\mathfrak{D}^{\perp}$ is a totally real distribution, and $\mathfrak{D}^{\theta}$ is a pointwise slant distribution whose slant function $\theta$ has values in $\left(0, \frac{\pi}{2}\right)$. Denote by $N_{T}, N_{\perp}$, and $N_{\theta}$ integrable submanifolds of $\mathfrak{D}^{T}, \mathfrak{D}^{\perp}$, and $\mathfrak{D}^{\theta}$, respectively. We call $M$ a pointwise $C R$-slant warped product if the induced metric $g$ on $M$ is a warped product metric of the form

$$
g=g_{B}+f^{2} g_{N_{\theta}},
$$

where $g_{B}$ is the metric of $B=N_{T} \times N_{\perp}, g_{N_{\theta}}$ is the metric on $N_{\theta}$, and $f$ is a positive function depending only on $B$. This warped product is called proper if the warping function $f$ is non-constant. In particular, if the slant function $\theta$ of $\mathfrak{D}^{\theta}$ is a constant in $\left(0, \frac{\pi}{2}\right)$, then $M$ is called a $C R$-slant warped product.

Notation 3.4. We simply denote the pointwise CR-slant warped product above by $\left(N_{T} \times N_{\perp}\right) \times_{f} N_{\theta}$, or by $N_{B} \times_{f} N_{\theta}$ with $B=N_{T} \times N_{\perp}$. 
Let $M=B \times_{f} N_{\theta}$ be a pointwise CR-slant warped product in $\tilde{M}$ with $B=$ $N_{T} \times N_{\perp}$. Then we have

$$
T M=\mathfrak{D}^{T} \oplus \mathfrak{D}^{\perp} \oplus \mathfrak{D}^{\theta}, \quad T^{\perp} M=J \mathfrak{D}^{\perp} \oplus F \mathfrak{D}^{\theta} \oplus \mu,
$$

where $\mu$ is a $J$-invariant subbundle of the normal bundle $T^{\perp} M$.

We make the following definition.

Definition 3.5. A pointwise CR-slant warped product $\left(N_{T} \times N_{\perp}\right) \times_{f} N_{\theta}$ in $\tilde{M}$ is called weakly $\mathfrak{D}^{\theta}$-totally geodesic if its second fundamental form $\sigma$ satisfies $\left\langle\sigma\left(\mathfrak{D}^{\theta}, \mathfrak{D}^{\theta}\right), J \mathfrak{D}^{\perp}\right\rangle=\{0\}$, i.e., $\sigma\left(\mathfrak{D}^{\theta}, \mathfrak{D}^{\theta}\right)$ has no component in $J \mathfrak{D}^{\perp}$.

We provide the following example of pointwise CR-slant warped product in $\mathbb{C}^{9}$ which is weakly $\mathfrak{D}^{\theta}$-totally geodesic.

Example 3.6. Let $\psi: \mathbb{R}^{5} \rightarrow \mathbb{C}^{9}$ be an isometric immersion given by

$$
\begin{aligned}
\psi(u, v, w, r, s)=( & u \cos r, u \cos s, w \cos r, w \cos s, u \sin r, \\
& u \sin s, r, w \sin r, w \sin s, v \cos r, v \cos s, w \sin r, \\
& w \sin s, v \sin r, v \sin s, s, w \cos r, w \cos s),
\end{aligned}
$$

with $u, v, w>0$. Then the tangent bundle $T M$ is spanned by

$$
\begin{aligned}
X_{1}= & (\cos r, \cos s, 0,0, \sin r, \sin s, 0,0,0,0,0,0,0,0,0,0,0,0), \\
X_{2}= & (0,0,0,0,0,0,0,0,0, \cos r, \cos s, 0,0, \sin r, \sin s, 0,0,0), \\
X_{3}= & (0,0, \cos r, \cos s, 0,0,0, \sin r, \sin s, \\
& \quad 0,0, \sin r, \sin s, 0,0,0, \cos r, \cos s), \\
X_{4}= & (-u \sin r, 0,-w \sin r, 0, u \cos r, 0,1, w \cos r, 0, \\
& \quad-v \sin r, 0, w \cos r, 0, v \cos r, 0,0,-w \sin r, 0), \\
X_{5}= & (0,-u \sin s, 0,-w \sin s, 0, u \cos s, 0,0, w \cos s, \\
& \quad 0,-v \sin s, 0, w \cos s, 0, v \cos s, 1,0,-w \sin s) .
\end{aligned}
$$

Then we find from 2.7 and 3.3 that

$$
\begin{aligned}
J X_{1}= & (0,0,0,0,0,0,0,0,0, \cos r, \cos s, 0,0, \sin r, \sin s, 0,0,0), \\
J X_{2}= & -(\cos r, \cos s, 0,0, \sin r, \sin s, 0,0,0,0,0,0,0,0,0,0,0,0), \\
J X_{3}= & (0,0,-\sin r,-\sin s, 0,0,0,-\cos r,-\cos s, \\
& \quad 0,0, \cos r, \cos s, 0,0,0, \sin r, \sin s), \\
J X_{4}= & (v \sin r, 0,-w \cos r, 0,-v \cos r, 0,0, w \sin r, 0, \\
& \quad-u \sin r, 0,-w \sin r, 0, u \cos r, 0,1, w \cos r, 0), \\
J X_{5}= & (0, v \sin s, 0,-w \cos s, 0,-v \cos s,-1,0, w \sin s, \\
& \quad 0,-u \sin s, 0,-w \sin s, 0, u \cos s, 0,0, w \cos s) .
\end{aligned}
$$

Since $J X_{3}$ is perpendicular to $T M, \mathfrak{D}^{\perp}=\operatorname{Span}\left\{X_{3}\right\}$ is totally real. Moreover, the distributions $\mathfrak{D}^{T}=\operatorname{Span}\left\{X_{1}, X_{2}\right\}$ and $\mathfrak{D}^{\theta}=\operatorname{Span}\left\{X_{4}, X_{5}\right\}$ are complex and proper pointwise slant with slant function $\theta=\cos ^{-1}\left(1 /\left(1+u^{2}+v^{2}+2 w^{2}\right)\right)$, respectively. 
Obviously, all the three distributions are integrable. Let $N_{T}, N_{\perp}$, and $N_{\theta}$ be the leaves of $\mathfrak{D}^{T}, \mathfrak{D}^{\perp}$, and $\mathfrak{D}^{\theta}$, respectively. Then the induced metric on the product manifold $N_{T} \times N_{\perp} \times N_{\theta}$ is the warped product metric:

$$
\begin{aligned}
g & =2\left(d u^{2}+d v^{2}\right)+4 d w^{2}+\left(1+u^{2}+v^{2}+2 w^{2}\right)\left(d r^{2}+d s^{2}\right) \\
& =g_{B}+f^{2} g_{N_{\theta}} .
\end{aligned}
$$

Hence, $M$ is a proper pointwise CR-slant warped product submanifold of $\mathbb{C}^{9}$ with warping function $f=\sqrt{1+u^{2}+v^{2}+2 w^{2}}$. We derive from (3.3) and (3.5) that

$$
\begin{aligned}
& \sigma\left(X_{i}, X_{j}\right)=0, \quad 1 \leq i, j \leq 3, \\
& \sigma\left(X_{1}, X_{4}\right)=\frac{-1}{1+u^{2}+v^{2}+2 w^{2}}\left(\left(1+v^{2}+2 w^{2}\right) \sin r, 0,-u w \sin r,\right. \\
& 0,-\left(1+v^{2}+2 w^{2}\right) \cos r, 0, u, u w \cos r, 0, \\
& -u v \sin r, 0, u w \cos r, 0, u v \cos r, 0,0,-u w \sin r, 0) \text {, } \\
& \sigma\left(X_{1}, X_{5}\right)=\frac{-1}{1+u^{2}+v^{2}+2 w^{2}}\left(0,\left(1+v^{2}+2 w^{2}\right) \sin s, 0,-u w \sin s,\right. \\
& 0,-\left(1+v^{2}+2 w^{2}\right) \cos s, 0,0, u w \cos s, 0, \\
& -u v \sin s, 0, u w \cos s, 0, u v \cos s, u, 0,-u w \sin s) \text {, } \\
& \sigma\left(X_{2}, X_{4}\right)=\frac{1}{1+u^{2}+v^{2}+2 w^{2}}(u v \sin r, 0, v w \sin r, 0,-u v \cos r, 0,-v, \\
& -v w \cos r, 0,-\left(1+u^{2}+2 w^{2}\right) \sin r, 0,-v w \cos r, \\
& \left.0,\left(1+u^{2}+2 w^{2}\right) \cos r, 0,0, v w \sin r, 0\right) \text {, } \\
& \sigma\left(X_{2}, X_{5}\right)=\frac{1}{1+u^{2}+v^{2}+2 w^{2}}(0, u v \sin s, 0, v w \sin s, 0,-u v \cos s, 0,0 \text {, } \\
& -v w \cos s, 0,-\left(1+u^{2}+2 w^{2}\right) \sin s, 0,-v w \cos s, \\
& \left.0,\left(1+u^{2}+2 w^{2}\right) \cos s,-v, 0, v w \sin s\right) \text {, } \\
& \sigma\left(X_{3}, X_{4}\right)=\frac{1}{1+u^{2}+v^{2}+2 w^{2}}\left(2 u w \sin r, 0,-\left(1+u^{2}+v^{2}\right) \sin r, 0,\right. \\
& -2 u w \cos r, 0,-2 w,\left(1+u^{2}+v^{2}\right) \cos r, 0,2 v w \sin r, 0, \\
& \left.\left(1+u^{2}+v^{2}\right) \cos r, 0,-2 v w \cos r, 0,0,-\left(1+u^{2}+v^{2}\right) \sin r, 0\right), \\
& \sigma\left(X_{3}, X_{5}\right)=\frac{1}{1+u^{2}+v^{2}+2 w^{2}}\left(0,2 u w \sin s, 0,-\left(1+u^{2}+v^{2}\right) \sin s, 0,\right. \\
& -2 u w \cos s, 0,0,\left(1+u^{2}+v^{2}\right) \cos s, 0,2 v w \sin s, 0, \\
& \left.\left(1+u^{2}+v^{2}\right) \cos s, 0,-2 v w \cos s,-2 w, 0,-\left(1+u^{2}+v^{2}\right) \sin s\right), \\
& \sigma\left(X_{4}, X_{4}\right)=-\frac{1}{2}(u \cos r,-u \cos s, w \cos r,-w \cos s, u \sin r,-u \sin s, \\
& 0, w \sin r,-w \sin s, v \cos r,-v \cos s, w \sin r,-w \sin s, \\
& v \sin r,-v \sin s, 0, w \cos r,-w \cos s), \\
& \sigma\left(X_{4}, X_{5}\right)=0, \quad \sigma\left(X_{5}, X_{5}\right)=-\sigma\left(X_{4}, X_{4}\right) \text {. }
\end{aligned}
$$


By applying (3.4) and (3.6), it is easy to verify that the pointwise CR-slant warped product defined by $\psi$ is weakly $\mathfrak{D}^{\theta}$-totally geodesic. Further, 3.5 and 3.6 imply that $N_{\theta}$ is not totally umbilical in $\mathbb{C}^{9}$.

\section{LEMMAS AND PROPOSITIONS}

In the following, we use the conventions that $X_{1}, Y_{1}$ are vector fields in $\mathfrak{D}^{T}$, $X_{2}, Y_{2}$ are vector fields in $\mathfrak{D}^{\perp}$, and $X_{3}, Y_{3}$ are vector fields in $\mathfrak{D}^{\theta}$.

Lemma 4.1. Let $M=B \times_{f} N_{\theta}$ be a pointwise CR-slant warped product in a Kaehler manifold $\tilde{M}$ with $B=N_{T} \times N_{\perp}$. Then we have:

(i) $\left\langle\sigma\left(X_{1}, Y_{1}\right), F X_{3}\right\rangle=0$,

(ii) $\left\langle\sigma\left(X_{1}, X_{3}\right), F Y_{3}\right\rangle=-J X_{1}(\ln f)\left\langle X_{3}, Y_{3}\right\rangle-X_{1}(\ln f)\left\langle X_{3}, P Y_{3}\right\rangle$, for any $X_{1}, Y_{1} \in \Gamma\left(\mathfrak{D}^{T}\right)$ and $X_{3}, Y_{3} \in \Gamma\left(\mathfrak{D}^{\theta}\right)$.

Proof. The first part of the lemma is easy and it can be derived by using 2.2, (1.1), and the orthogonality of vector fields. On the other hand, we have

$$
\begin{aligned}
\left\langle\sigma\left(X_{1}, X_{3}\right), F Y_{3}\right\rangle & =\left\langle\tilde{\nabla}_{X_{3}} X_{1}, J Y_{3}-P Y_{3}\right\rangle \\
& =-\left\langle\tilde{\nabla}_{X_{3}} J X_{1}, Y_{3}\right\rangle-\left\langle\tilde{\nabla}_{X_{3}} X_{1}, P Y_{3}\right\rangle,
\end{aligned}
$$

for any $X_{1} \in \Gamma\left(\mathfrak{D}^{T}\right)$ and $X_{3}, Y_{3} \in \Gamma\left(\mathfrak{D}^{\theta}\right)$. Therefore, we obtain the required result from 1.1 .

Lemma 4.2. Let $M=B \times_{f} N_{\theta}$ be a pointwise CR-slant warped product in a Kaehler manifold $\tilde{M}$ with $B=N_{T} \times N_{\perp}$. Then

(i) $\left\langle\sigma\left(X_{1}, X_{3}\right), J X_{2}\right\rangle=0$,

(ii) $\left\langle\sigma\left(X_{1}, X_{2}\right), F X_{3}\right\rangle=0$,

for any $X_{1} \in \Gamma\left(\mathfrak{D}^{T}\right), X_{2} \in \Gamma\left(\mathfrak{D}^{\perp}\right)$, and $X_{3} \in \Gamma\left(\mathfrak{D}^{\theta}\right)$.

Proof. From 2.2 and (2.1), we have

$$
\left\langle\sigma\left(X_{1}, X_{3}\right), J X_{2}\right\rangle=\left\langle\tilde{\nabla}_{X_{3}} X_{1}, J X_{2}\right\rangle=-\left\langle\tilde{\nabla}_{X_{3}} J X_{1}, X_{2}\right\rangle
$$

Using (1.1) and the orthogonality of vector fields, we have (i). In a similar way, we obtain (ii).

Lemma 4.3. Let $M=B \times_{f} N_{\theta}$ be a pointwise CR-slant warped product in a Kaehler manifold $\tilde{M}$ with $B=N_{T} \times N_{\perp}$. Then we have:

(i) $\left\langle\sigma\left(X_{2}, Y_{2}\right), F X_{3}\right\rangle=\left\langle\sigma\left(X_{2}, X_{3}\right), J Y_{2}\right\rangle$,

(ii) $\left\langle\sigma\left(X_{3}, Y_{3}\right), J X_{2}\right\rangle-\left\langle\sigma\left(X_{2}, X_{3}\right), F Y_{3}\right\rangle=X_{2}(\ln f)\left\langle X_{3}, P Y_{3}\right\rangle$,

for any $X_{2}, Y_{2} \in \Gamma\left(\mathfrak{D}^{\perp}\right)$ and $X_{3}, Y_{3} \in \Gamma\left(\mathfrak{D}^{\theta}\right)$.

Proof. The first part follows easily from (2.2), (2.1), (1.1), and (2.3). For the second part, we have

$$
\left\langle\sigma\left(X_{3}, Y_{3}\right), J X_{2}\right\rangle=\left\langle\tilde{\nabla}_{X_{3}} Y_{3}, J X_{2}\right\rangle=-\left\langle\tilde{\nabla}_{X_{3}} P Y_{3}, X_{2}\right\rangle-\left\langle\tilde{\nabla}_{X_{3}} F Y_{3}, X_{2}\right\rangle .
$$

Using (1.1) and (2.1), we derive

$$
\left\langle\sigma\left(X_{3}, Y_{3}\right), J X_{2}\right\rangle=X_{2}(\ln f)\left\langle X_{3}, P Y_{3}\right\rangle-\left\langle J \tilde{\nabla}_{X_{3}} F Y_{3}, J X_{2}\right\rangle .
$$


On the other hand, applying 2.4 and 2.6, we have

$$
\begin{aligned}
\left\langle J \tilde{\nabla}_{X_{3}} F Y_{3}, J X_{2}\right\rangle= & \left\langle\tilde{\nabla}_{X_{3}} t F Y_{3}, J X_{2}\right\rangle+\left\langle\tilde{\nabla}_{X_{3}} f F Y_{3}, J X_{2}\right\rangle \\
= & -\sin ^{2} \theta\left\langle\tilde{\nabla}_{X_{3}} Y_{3}, J X_{2}\right\rangle-\sin (2 \theta) X_{3}(\theta)\left\langle Y_{3}, J X_{2}\right\rangle \\
& -\left\langle\tilde{\nabla}_{X_{3}} F P Y_{3}, J X_{2}\right\rangle .
\end{aligned}
$$

Again using 2.6, we derive

$$
\begin{aligned}
\left\langle J \tilde{\nabla}_{X_{3}} F Y_{3}, J X_{2}\right\rangle= & -\sin ^{2} \theta\left\langle\sigma\left(X_{3}, Y_{3}\right), J X_{2}\right\rangle+\left\langle\tilde{\nabla}_{X_{3}} t F P Y_{3}, X_{2}\right\rangle \\
& +\left\langle\tilde{\nabla}_{X_{3}} f F P Y_{3}, X_{2}\right\rangle \\
= & -\sin ^{2} \theta\left\langle\sigma\left(X_{3}, Y_{3}\right), J X_{2}\right\rangle-\sin ^{2} \theta\left\langle\tilde{\nabla}_{X_{3}} P Y_{3}, X_{2}\right\rangle \\
& -\sin (2 \theta) X_{3}(\theta)\left\langle P Y_{3}, X_{2}\right\rangle-\left\langle\tilde{\nabla}_{X_{3}} F P^{2} Y_{3}, X_{2}\right\rangle .
\end{aligned}
$$

Then (1.1) and 2.5 yield with the orthogonality of vector fields that

$$
\begin{aligned}
\left\langle J \tilde{\nabla}_{X_{3}} F Y_{3}, J X_{2}\right\rangle= & -\sin ^{2} \theta\left\langle\sigma\left(X_{3}, Y_{3}\right), J X_{2}\right\rangle+X_{2}(\ln f) \sin ^{2} \theta\left\langle X_{3}, P Y_{3}\right\rangle \\
& +\cos ^{2} \theta\left\langle\tilde{\nabla}_{X_{3}} F Y_{3}, X_{2}\right\rangle-\sin (2 \theta) X_{3}(\theta)\left\langle F Y_{3}, X_{2}\right\rangle .
\end{aligned}
$$

Hence (ii) follows from 4.1 and 4.2 by using the orthogonality of vector fields. This completes the proof of the lemma.

Definition 4.4. A pointwise CR-slant warped product $\left(N_{T} \times N_{\perp}\right) \times_{f} N_{\theta}$ is called $\mathfrak{D}^{1} \oplus \mathfrak{D}^{2}$-mixed totally geodesic if its second fundamental form $\sigma$ satisfies

$$
\sigma\left(\mathfrak{D}^{1}, \mathfrak{D}^{2}\right)=\{0\}
$$

where $\mathfrak{D}^{1}$ and $\mathfrak{D}^{2}$ are distributions from $\left\{\mathfrak{D}^{T}, \mathfrak{D}^{\perp}, \mathfrak{D}^{\theta}\right\}$.

Proposition 4.5. Let $M=B \times_{f} N_{\theta}$ be a pointwise CR-slant warped product in a Kaehler manifold $\tilde{M}$ with $B=N_{T} \times N_{\perp}$. If $M$ is $\mathfrak{D}^{T} \oplus \mathfrak{D}^{\theta}$-mixed totally geodesic, then the warping function $f$ depends only on $N_{\perp}$.

Proof. From Lemma 4.1(ii), we have

$$
\left\langle\sigma\left(J X_{1}, X_{3}\right), F Y_{3}\right\rangle=X_{1}(\ln f)\left\langle X_{3}, Y_{3}\right\rangle+J X_{1}(\ln f)\left\langle P X_{3}, Y_{3}\right\rangle .
$$

Replacing $X_{3}$ by $P X_{3}$ and using (2.5), we get

$$
\left\langle\sigma\left(J X_{1}, P X_{3}\right), F Y_{3}\right\rangle=X_{1}(\ln f)\left\langle P X_{3}, Y_{3}\right\rangle-J X_{1}(\ln f) \cos ^{2} \theta\left\langle X_{3}, Y_{3}\right\rangle .
$$

Hence, from Lemma 4.1 (ii) and (3.6), we derive

$$
\left\langle\sigma\left(X_{1}, X_{3}\right), F Y_{3}\right\rangle-\left\langle\sigma\left(J X_{1}, P X_{3}\right), F Y_{3}\right\rangle=-J X_{1}(\ln f) \sin ^{2} \theta\left\langle X_{3}, Y_{3}\right\rangle .
$$

Hence, if $M$ is $\mathfrak{D}^{T} \oplus \mathfrak{D}^{\theta}$-mixed totally geodesic, the result follows from 4.3 ).

Proposition 4.6. Let $M=B \times_{f} N_{\theta}$ be a pointwise CR-slant warped product in a Kaehler manifold $\tilde{M}$ with $B=N_{T} \times N_{\perp}$. If $M$ is $\mathfrak{D}^{\perp} \oplus \mathfrak{D}^{\theta}$-mixed totally geodesic, then the warping function $f$ depends only on $N_{T}$. 
Proof. Using the polarization identity in Lemma 4.3 (ii) for vector fields $X_{3}, Y_{3} \in$ $\Gamma\left(\mathfrak{D}^{\theta}\right)$, we find

$$
\left\langle\sigma\left(X_{3}, Y_{3}\right), J X_{2}\right\rangle=-X_{2}(\ln f)\left\langle X_{3}, P Y_{3}\right\rangle+\left\langle\sigma\left(Y_{3}, X_{2}\right), F X_{3}\right\rangle .
$$

Thus we find from Lemma 4.3 (ii) and 4.4 that

$$
\left\langle\sigma\left(X_{2}, Y_{3}\right), F X_{3}\right\rangle-\left\langle\sigma\left(X_{2}, X_{3}\right), F Y_{3}\right\rangle=X_{2}(\ln f)\left\langle X_{3}, P Y_{3}\right\rangle .
$$

Now, replacing $X_{3}$ by $P X_{3}$, we obtain

$$
\left\langle\sigma\left(X_{2}, Y_{3}\right), F P X_{3}\right\rangle-\left\langle\sigma\left(X_{2}, P X_{3}\right), F Y_{3}\right\rangle=X_{2}(\ln f) \cos ^{2} \theta\left\langle X_{3}, Y_{3}\right\rangle .
$$

Thus, if $M$ is $\mathfrak{D}^{\perp} \oplus \mathfrak{D}^{\theta}$-mixed totally geodesic, then 4.5) yields the result.

Remark 4.7. Propositions 4.5 and 4.6 imply that a pointwise CR-slant warped product $\left(N_{T} \times N_{\perp}\right) \times_{f} N_{\theta}$ is a Riemannian product if it is $\mathfrak{D}^{T} \oplus \mathfrak{D}^{\theta}$-mixed totally geodesic and $\mathfrak{D}^{\perp} \oplus \mathfrak{D}^{\theta}$-mixed totally geodesic.

\section{The MAin theorem: A SharP inequality For $\|\sigma\|^{2}$}

Consider a pointwise CR-slant warped product $M=B \times_{f} N_{\theta}$ in a Kaehler manifold $\tilde{M}$ with $\operatorname{dim} M=n, \operatorname{dim}_{\mathbb{R}} \tilde{M}=2 m$, and $B=N_{T} \times N_{\perp}$. We choose a local orthonormal frame $\left\{e_{1}, \ldots, e_{n}\right\}$ of $T M$ such that

$$
\begin{aligned}
& \mathfrak{D}^{\perp}= \operatorname{Span}\left\{e_{1}=e_{1}, \ldots, e_{q}\right\}, \\
& \mathfrak{D}^{T}=\operatorname{Span}\left\{e_{q+1}=\hat{e}_{1}, \ldots, e_{q+p}=\hat{e}_{p}, \ldots, e_{q+p+1}=\hat{e}_{p+1}=J \hat{e}_{1},\right. \\
&\left.\quad \ldots, e_{q+2 p}=\hat{e}_{2 p}=J \hat{e}_{p}\right\} \\
& \mathfrak{D}^{\theta}=\operatorname{Span}\left\{e_{2 p+q+1}=e_{1}^{*}, \ldots, e_{2 p+q+s}=e_{s}^{*},\right. \\
&\left.e_{2 p+q+s+1}=\sec \theta P e_{1}^{*}, \ldots, e_{n}=\sec \theta P e_{s}^{*}\right\},
\end{aligned}
$$

with $q=\operatorname{dim} N_{\perp}, p=\frac{1}{2} \operatorname{dim} N_{T}$, and $s=\frac{1}{2} \operatorname{dim} N_{\theta}$. Also, we choose a local orthonormal frame $\left\{E_{1}, \ldots, E_{2 m-n}\right\}$ of the normal bundles of $T^{\perp} M$ such that

$$
\begin{aligned}
J \mathfrak{D}^{\perp}= & \operatorname{Span}\left\{E_{1}=J e_{1}, \ldots, E_{q}=J e_{q}\right\}, \\
F\left(\mathfrak{D}^{\theta}\right)= & \operatorname{Span}\left\{E_{q+1}=\csc \theta F e_{1}^{*}, \ldots, E_{q+s}=\csc \theta F e_{s}^{*},\right. \\
& \left.E_{q+s+1}=\csc \theta \sec \theta F P e_{1}^{*}, \ldots, E_{q+2 s}=\csc \theta \sec \theta F P e_{s}^{*}\right\} \\
\mu= & \operatorname{Span}\left\{E_{q+2 s+1}, \ldots, E_{2 m-n}\right\},
\end{aligned}
$$

where $\mu$ is a $J$-invariant normal subbundle of $T^{\perp} M$.

The following theorem gives a sharp inequality involving the norm $\|\sigma\|$ of the second fundamental form for CR-slant warped products in any Kaehler manifold.

Theorem 5.1. Let $M=B \times_{f} N_{\theta}$ be a pointwise CR-slant warped product in a Kaehler manifold $\tilde{M}$ with $B=N_{T} \times N_{\perp}$. If $M$ is weakly $\mathfrak{D}^{\theta}$-totally geodesic, then

(i) The squared norm $\|\sigma\|^{2}$ of the second fundamental form of $M$ satisfies

$$
\|\sigma\|^{2} \geq 4 s\left[\left(\csc ^{2} \theta+\cot ^{2} \theta\right)\left\|\nabla^{T}(\ln f)\right\|^{2}+\cot ^{2} \theta\left\|\nabla^{\perp}(\ln f)\right\|^{2}\right],
$$

where $\nabla^{T}(\ln f)$ and $\nabla^{\perp}(\ln f)$ denote the gradient components of $\ln f$ along $N_{T}$ and $N_{\perp}$, respectively, and $s=\frac{1}{2} \operatorname{dim} N_{\theta}$. 
(ii) The equality sign in (5.1) holds identically if and only if B is totally geodesic and $N_{\theta}$ is totally umbilical in $\tilde{M}$.

(iii) If the warping function $f$ in $B \times_{f} N_{\theta}$ is non-constant, then at least one of $\mathfrak{D}^{T} \oplus \mathfrak{D}^{\theta}$ and $\mathfrak{D}^{\perp} \oplus \mathfrak{D}^{\theta}$ is non-mixed totally geodesic in $\tilde{M}$.

Proof. From the definition of $\sigma$, we have

$$
\|\sigma\|^{2}=\sum_{i, j=1}^{n}\left\langle\sigma\left(e_{i}, e_{j}\right), \sigma\left(e_{i}, e_{j}\right)\right\rangle=\sum_{r=n+1}^{2 m-n} \sum_{i, j=1}^{n}\left(\left\langle\sigma\left(e_{i}, e_{j}\right), E_{r}\right\rangle\right)^{2},
$$

where $\left\{e_{n+1}, \ldots, e_{2 m-n}\right\}$ is a local orthonormal frame of the normal bundle. From (3.2), the above relation takes the form

$$
\begin{aligned}
\|\sigma\|^{2}= & \sum_{r=1}^{q} \sum_{i, j=1}^{n}\left(\left\langle\sigma\left(e_{i}, e_{j}\right), J e_{r}\right\rangle\right)^{2}+\sum_{r=q+1}^{q+2 s} \sum_{i, j=1}^{n}\left(\left\langle\sigma\left(e_{i}, e_{j}\right), E_{r}\right\rangle\right)^{2} \\
& +\sum_{r=q+2 s+1}^{2 m-n} \sum_{i, j=1}^{n}\left(\left\langle\sigma\left(e_{i}, e_{j}\right), E_{r}\right\rangle\right)^{2} .
\end{aligned}
$$

Leaving the last $\mu$-components term in $(5.2)$ and using the frame fields given above, we find

$$
\begin{aligned}
\|\sigma\|^{2} & \geq \sum_{r=1}^{q} \sum_{i, j=1}^{2 p}\left(\left\langle\sigma\left(\hat{e}_{i}, \hat{e}_{j}\right), J e_{r}\right\rangle\right)^{2}+2 \sum_{r=1}^{q} \sum_{i, j=1}^{2 p} \sum_{j=1}^{q}\left(\left\langle\sigma\left(\hat{e}_{i}, e_{j}\right), J e_{r}\right\rangle\right)^{2} \\
& +2 \sum_{r=1}^{q} \sum_{i, j=1}^{2 p} \sum_{j=1}^{2 s}\left(\left\langle\sigma\left(\hat{e}_{i}, e_{j}^{*}\right), J e_{r}\right\rangle\right)^{2}+\sum_{r=1}^{q} \sum_{i, j=1}^{q}\left(\left\langle\sigma\left(e_{i}, e_{j}\right), J e_{r}\right\rangle\right)^{2} \\
& +2 \sum_{r=1}^{q} \sum_{i=1}^{q} \sum_{j=1}^{2 s}\left(\left\langle\sigma\left(e_{i}, e_{j}^{*}\right), J e_{r}\right\rangle\right)^{2}+\sum_{r=1}^{q} \sum_{i, j=1}^{2 s}\left(\left\langle\sigma\left(e_{i}^{*}, e_{j}^{*}\right), J e_{r}\right\rangle\right)^{2} \\
& +\csc ^{2} \theta \sum_{r=1}^{s} \sum_{i, j=1}^{2 p}\left[\left(\left\langle\sigma\left(\hat{e}_{i}, \hat{e}_{j}\right), F e_{r}^{*}\right\rangle\right)^{2}+\sec ^{2} \theta\left(\left\langle\sigma\left(\hat{e}_{i}, \hat{e}_{j}\right), F P e_{r}^{*}\right\rangle\right)^{2}\right] \\
& +2 \csc ^{2} \theta \sum_{r=1}^{s} \sum_{i=1}^{2 p} \sum_{j=1}^{q}\left[\left(\left\langle\sigma\left(\hat{e}_{i}, e_{j}\right), F e_{r}^{*}\right\rangle\right)^{2}+\sec ^{2} \theta\left(\left\langle\sigma\left(\hat{e}_{i}, e_{j}\right), F P e_{r}^{*}\right\rangle\right)^{2}\right] \\
& +2 \csc ^{2} \theta \sum_{r=1}^{s} \sum_{i=1}^{2 p} \sum_{j=1}^{2 s}\left[\left(\left\langle\sigma\left(\hat{e}_{i}, e_{j}^{*}\right), F e_{r}^{*}\right\rangle\right)^{2}+\sec ^{2} \theta\left(\left\langle\sigma\left(\hat{e}_{i}, e_{j}^{*}\right), F P e_{r}^{*}\right\rangle\right)^{2}\right] \\
& +\csc ^{2} \theta \sum_{r=1}^{s} \sum_{i, j=1}^{q}\left[\left(\left\langle\sigma\left(e_{i}, e_{j}\right), F e_{r}^{*}\right\rangle\right)^{2}+\sec ^{2} \theta\left(\left\langle\sigma\left(e_{i}, e_{j}\right), F P e_{r}^{*}\right\rangle\right)^{2}\right] \\
& +\csc ^{2} \theta \sum_{r=1}^{s} \sum_{i, j=1}^{2 s}\left[\left(\left\langle\sigma\left(e_{i}^{*}, e_{j}^{*}\right), F e_{r}^{*}\right\rangle\right)^{2}+\sec ^{2} \theta\left(\left\langle\sigma\left(e_{i}^{*}, e_{j}^{*}\right), F P e_{r}^{*}\right\rangle\right)^{2}\right]
\end{aligned}
$$




$$
+2 \csc ^{2} \theta \sum_{r=1}^{s} \sum_{i=1}^{q} \sum_{j=1}^{2 s}\left[\left(\left\langle\sigma\left(e_{i}, e_{j}^{*}\right), F e_{r}^{*}\right\rangle\right)^{2}+\sec ^{2} \theta\left(\left\langle\sigma\left(e_{i}, e_{j}^{*}\right), F P e_{r}^{*}\right\rangle\right)^{2}\right] .
$$

There is no relation for warped products for the first, second, fourth, and eleventh terms in (5.3). So, we leave these positive terms. Further, using Lemmas 4.1 4.3 with the hypothesis of the theorem and the orthogonality of vector fields, we obtain

$$
\begin{aligned}
\|\sigma\|^{2} \geq & 4 s \csc ^{2} \theta \sum_{r=1}^{p}\left[\left(-J e_{i}(\ln f)\right)^{2}+\left(e_{i}(\ln f)\right)^{2}\right] \\
& +4 s \cot ^{2} \theta \sum_{r=1}^{p}\left[\left(-J e_{i}(\ln f)\right)^{2}+\left(e_{i}(\ln f)\right)^{2}\right]+4 s \cot ^{2} \theta \sum_{r=1}^{q}\left(e_{i}(\ln f)\right)^{2} \\
= & 4 s\left(\csc ^{2} \theta+\cot ^{2} \theta\right) \sum_{r=1}^{2 p}\left[\left(e_{i}(\ln f)\right)^{2}+4 s \cot ^{2} \theta \sum_{r=1}^{q}\left(e_{i}(\ln f)\right)^{2}\right]
\end{aligned}
$$

which gives the inequality (5.1). For the equality case, from the omitted terms and the vanishing terms in $(5.2)$ and $(5.3)$, we find

$$
\sigma\left(\mathfrak{D}^{\theta}, \mathfrak{D}^{\theta}\right)=0, \quad \sigma\left(\mathfrak{D}^{T}, \mathfrak{D}^{T}\right)=0, \quad \sigma\left(\mathfrak{D}^{\perp}, \mathfrak{D}^{\perp}\right)=0, \quad \sigma\left(\mathfrak{D}^{T}, \mathfrak{D}^{\perp}\right)=0 .
$$

We also have

$$
\sigma\left(\mathfrak{D}^{T}, \mathfrak{D}^{\theta}\right) \subset F \mathfrak{D}^{\theta}, \quad \sigma\left(\mathfrak{D}^{\perp}, \mathfrak{D}^{\theta}\right) \subset F \mathfrak{D}^{\theta} .
$$

Since $B$ is totally geodesic in $M$ (cf. e.g. [1, 6]), using this fact together with (5.4), we know that $B$ is totally geodesic in $\tilde{M}$. Also, since $N_{\theta}$ is totally umbilical in $M$, using this fact together with (5.5), we conclude that $N_{\theta}$ is totally umbilical in $\tilde{M}$. The converse is direct to verify. This gives statement (ii).

Statement (iii) follows from Remark 4.7 .

\section{Some Applications}

The following results follow easily from Theorem 5.1

Theorem 6.1 ([6]). If $N_{T} \times_{f} N_{\perp}$ is a CR-warped product in a Kaehler manifold $\tilde{M}$, then we have:

$$
\|\sigma\|^{2} \geq 2 p\left\|\nabla^{T}(\ln f)\right\|^{2},
$$

where $p=\operatorname{dim} N_{\perp}$ and $\nabla^{T}(\ln f)$ denotes the gradient of $\ln f$ on $N_{T}$. Moreover, if the equality sign in (6.1) holds identically, then $N_{T}$ is totally geodesic and $N_{\perp}$ is totally umbilical in $M$.

Theorem $6.2([22])$. If $N_{T} \times_{f} N_{\theta}$ is a warped product pointwise semi-slant submanifold of a Kaehler manifold $\tilde{M}$, then we have

$$
\|\sigma\|^{2} \geq 4 s\left(\csc ^{2} \theta+\cot ^{2} \theta\right)\left\|\nabla^{T}(\ln f)\right\|^{2}, \quad \operatorname{dim} N_{\theta}=2 s .
$$

Furthermore, if the equality sign in 6.2 holds identically, then $N_{T}$ is totally geodesic and $N_{\theta}$ is totally umbilical in $M$.

If $\operatorname{dim} N_{T}=0$, then Theorem 5.1 implies the following. 
Theorem $6.3([23])$. If $N_{\perp} \times_{f} N_{\theta}$ is a $\mathfrak{D}^{\perp} \oplus \mathfrak{D}^{\theta}$-mixed totally geodesic warped product pointwise hemi-slant (pseudo-slant) submanifold of a Kaehler manifold $\tilde{M}$, then its second fundamental form $\sigma$ satisfies

$$
\|\sigma\|^{2} \geq 2 s \cos ^{2} \theta\left\|\nabla^{\perp}(\ln f)\right\|^{2}, \quad \operatorname{dim} N_{\theta}=2 s,
$$

where $\nabla^{\perp}(\ln f)$ denotes the gradient of $\ln f$ on $N_{\perp}$.

Remark 6.4. Theorem 5.1 improves Theorem 6.3 since if $M$ is $\mathfrak{D}^{\perp} \oplus \mathfrak{D}^{\theta}$-mixed totally geodesic, then $M$ is Riemannian product of $N_{\perp}$ and $N_{\theta}$ by Remark 4.7 . which is the original statement of Corollary 4.5 of [26]. Thus, Theorem 5.1 implies that the inequality 6.3 , obtained in [23], is not sharp.

\section{REFERENCES}

[1] R. L. Bishop and B. O'Neill, Manifolds of negative curvature, Trans. Amer. Math. Soc. 145 (1969), 1-49. MR 0251664

[2] B.-Y. Chen, CR-submanifolds of a Kaehler manifold. I, J. Differential Geometry 16 (1981), no. 2, 305-322. MR 0638795.

[3] B.-Y. Chen, CR-submanifolds of a Kaehler manifold. II, J. Differential Geometry 16 (1981), no. 3, 493-509 (1982). MR 0654640

[4] B.-Y. Chen, Slant immersions, Bull. Austral. Math. Soc. 41 (1990), no. 1, 135-147. MR 1043974

[5] B.-Y. Chen, Geometry of slant submanifolds, Katholieke Universiteit Leuven, Louvain, 1990. MR 1099374

[6] B.-Y. Chen, Geometry of warped product CR-submanifolds in Kaehler manifolds, Monatsh. Math. 133 (2001), no. 3, 177-195. MR 1861136.

[7] B.-Y. Chen, Geometry of warped product CR-submanifolds in Kaehler manifolds. II, Monatsh. Math. 134 (2001), no. 2, 103-119. MR 1878074

[8] B.-Y. Chen, Another general inequality for CR-warped products in complex space forms, Hokkaido Math. J. 32 (2003), no. 2, 415-444. MR 1996288

[9] B.-Y. Chen, CR-warped products in complex projective spaces with compact holomorphic factor, Monatsh. Math. 141 (2004), no. 3, 177-186. MR 2042209

[10] B.-Y. Chen, Pseudo-Riemannian geometry, $\delta$-invariants and applications, World Scientific Publishing, Hackensack, NJ, 2011. MR 2799371

[11] B.-Y. Chen, Geometry of warped product submanifolds: a survey, J. Adv. Math. Stud. 6 (2013), no. 2, 1-43. MR 3136561

[12] B.-Y. Chen, Differential geometry of warped product manifolds and submanifolds, World Scientific Publishing, Hackensack, NJ, 2017. MR 3699316

[13] B.-Y. Chen and O. J. Garay, Pointwise slant submanifolds in almost Hermitian manifolds, Turkish J. Math. 36 (2012), no. 4, 630-640. MR 2993593

[14] B.-Y. Chen and K. Ogiue, On totally real submanifolds, Trans. Amer. Math. Soc. 193 (1974), 257-266. MR 0346708

[15] B.-Y. Chen and S. Uddin, Warped product pointwise bi-slant submanifolds of Kaehler manifolds, Publ. Math. Debrecen 92 (2018), no. 1-2, 183-199. MR 3764086.

[16] F. Etayo, On quasi-slant submanifolds of an almost Hermitian manifold, Publ. Math. Debrecen 53 (1998), no. 1-2, 217-223. MR 1661038 
[17] V. A. Khan and K. A. Khan, Generic warped product submanifolds in nearly Kaehler manifolds, Beiträge Algebra Geom. 50 (2009), no. 2, 337-352. MR 2572004

[18] I. Mihai, Contact CR-warped product submanifolds in Sasakian space forms, Geom. Dedicata 109 (2004), 165-173. MR 2114073.

[19] K.-S. Park, Pointwise almost $h$-semi-slant submanifolds, Internat. J. Math. 26 (2015), no. 12, 1550099, 26 pp. MR 3432530

[20] B. Sahin, Nonexistence of warped product semi-slant submanifolds of Kaehler manifolds, Geom. Dedicata 117 (2006), 195-202. MR 2231167

[21] B. Sahin, Warped product submanifolds of Kaehler manifolds with a slant factor, Ann. Polon. Math. 95 (2009), no. 3, 207-226. MR 2491378

[22] B. Sahin, Warped product pointwise semi-slant submanifolds of Kähler manifolds, Port. Math. 70 (2013), no. 3, 251-268. MR 3138091

[23] S. K. Srivastava and A. Sharma, Pointwise pseudo-slant warped product submanifolds in a Kähler manifold, Mediterr. J. Math. 14 (2017), no. 1, Paper No. 20, 18 pp. MR 3590362

[24] S. Uddin and A. Y. M. Chi, Warped product pseudo-slant submanifolds of nearly Kaehler manifolds, An. Ştiint. Univ. "Ovidius" Constanţa Ser. Mat. 19 (2011), no. 3, 195-204. MR 2879098

[25] S. Uddin, B.-Y. Chen and F. R. Al-Solamy, Warped product bi-slant immersions in Kaehler manifolds, Mediterr. J. Math. 14 (2017), no. 2, Paper No. 95, 11 pp. MR 3624900.

[26] S. Uddin and M. S. Stanković, Warped product submanifolds of Kaehler manifolds with pointwise slant fiber, Filomat 32 (2018), no. 1, 35-44. MR 3762248

[27] K. Yano and M. Kon, Structures on manifolds, Series in Pure Mathematics, 3, World Scientific Publishing, Singapore, 1984. MR 0794310.

\author{
Bang-Yen Chen ${ }^{\bowtie}$ \\ Department of Mathematics, Michigan State University, 619 Red Cedar Road, East Lansing, \\ Michigan 48824-1027, U.S.A. \\ bychen@math.msu.edu \\ Siraj Uddin \\ Department of Mathematics, Faculty of Science, King Abdulaziz University, 21589 Jeddah, \\ Saudi Arabia \\ siraj.ch@gmail.com \\ Falleh R. Al-Solamy \\ Department of Mathematics, Faculty of Science, King Abdulaziz University, 21589 Jeddah, \\ Saudi Arabia \\ falleh@hotmail.com
}

Received: April 17, 2019

Accepted: September 15, 2019 